GA-A24087

\title{
ADVANCED TOKAMAK OPERATION USING THE DIII-D PLASMA CONTROL SYSTEM
}

\author{
by \\ D.A. HUMPHREYS, J.R. FERRON, A.M. GAROFALO, A.W. HYATT, \\ T.C. JERNIGAN, R.D. JOHNSON, R.J. LA HAYE, J.A. LEUER, \\ M. OKABAYASHI, B.G. PENAFLOR, J.T. SCOVILLE, E.J. STRAIT, \\ M.L. WALKER, and D.G. WHYTE
}


DISCLAIMER

This report was prepared as an account of work sponsored by an agency of the United States Government. Neither the United States Government nor any agency thereof, nor any of their employees, makes any warranty, express or implied, or assumes any legal liability or responsibility for the accuracy, completeness, or usefulness of any information, apparatus, product, or process disclosed, or represents that its use would not infringe privately owned rights.

Reference herein to any specific commercial product, process, or service by trade name, trademark, manufacturer, or otherwise, does not necessarily constitute or imply its endorsement, recommendation, or favoring by the United States Government or any agency thereof. The views and opinions of authors expressed herein do not necessarily state or reflect those of the United States Government or any agency thereof. 


\title{
ADVANCED TOKAMAK OPERATION USING THE DIII-D PLASMA CONTROL SYSTEM
}

by

D.A. HUMPHREYS, J.R. FERRON, A.M. GAROFALO, † A.W. HYATT, T.C. JERNIGAN, ${ }^{\ddagger}$ R.D. JOHNSON, R.J. LA HAYE, J.A. LEUER, M. OKABAYASHI, ${ }^{\ominus}$ B.G. PENAFLOR, J.T. SCOVILLE, E.J. STRAIT, M.L. WALKER, and D.G. WHYTE ${ }^{\Delta}$

\author{
†Columbia University \\ Ғoak Ridge NationalLaboratory \\ $\checkmark$ Princeton Plasma Physics Laboratory \\ $\Delta$ University of California, San Diego
}

This is a preprint of a paper presented at the 22nd Symposium on Fusion Technology, September 9-13, 2002, in Helsinki, Finland, and to be published in Fusion Engineering and Design.

\author{
Work supported by \\ the U.S. Department of Energy \\ under Contracts DE-AC03-99ER54463, DE-AC05-000R22725, \\ DE-AC02-76CH03073, and Grants DE-FG02-89ER53297, \\ and DE-FG03-95ER54294
}

GA PROJECT 30033

OCTOBER 2002 


\section{ABSTRACT}

The principal focus of experimental operations in the DIII-D tokamak is the advanced tokamak (AT) [1] regime to achieve, which requires highly integrated and flexible plasma control. In a high performance advanced tokamak, accurate regulation of the plasma boundary, internal profiles, pumping, fueling, and heating must be well coordinated with MHD control action to stabilize such instabilities as tearing modes and resistive wall modes. Sophisticated monitors of the operational regime must provide detection of off-normal conditions and trigger appropriate safety responses with acceptable levels of reliability. Many of these capabilities are presently implemented in the DIII-D plasma control system (PCS), and are now in frequent or routine operational use. The present work describes recent development, implementation, and operational experience with AT regime control elements for equilibrium control, MHD suppression, and off-normal event detection and response. 


\section{INTRODUCTION}

A central characteristic of AT plasma regimes is the extreme shapes which must be accessed and the high degree of accuracy with which they must be regulated. The DIII-D isoflux shape control system in concert with the real-time EFIT (RTEFIT) equilibrium reconstruction algorithm [2] enables creation of a wide range of shape characteristics including highly indented beans, double-null, upper/lower single-null, limited, high triangularity and high squareness, all at high elongations.

This precise shape and position control has been used to enable active suppression of MHD instabilities. The DIII-D PCS can control the relative location of gyrotron ECCD deposition and neoclassical tearing mode (NTM) islands, suppressing the modes by replacing the "missing" bootstrap current that characterizes the instability. PCS algorithms for feedback control of nonaxisymmetric radial field coil currents based on measurements of $n=1$ magnetic signals can stabilize the resistive wall mode (RWM) beyond the no-wall $\beta$-limit.

Because AT plasmas operate close to stability limits in order to yield high efficiencies, offnormal events can conceivably produce large excursions from the operating point and result in uncontrollable instability. Algorithms have been implemented in the PCS to detect the impending onset of such instabilities and take corrective or mitigating action. The use of such monitors has allowed a reduction in total neutrons produced during a DIII-D high-performance run campaign, and allows triggering of high-pressure gas injection to almost completely mitigate disruption effects.

Finally, the PCS has also actuated the ECCD system and modified the current profile, offering the hope of actively regulating the performance and stability of AT plasmas. 


\section{EQUILIBRIUM AND PROFILE CONTROL FOR AT}

Off-axis electron cyclotron current drive (ECCD) has been applied in DIII-D to affect the safety factor $(q)$ profile as a first step toward current profile regulation. Figure 1(a) shows a simulation of the effect of applying 2.5 MW of highly localized ECCD at $\rho=0.4$. The simulation shows an increase in the axis safety factor $q_{0}$ from its initial value of $\sim 3.5$ to a maximum value of $\sim 4.5$ in $\sim 1.0 \mathrm{~s}$. Figure 1(b) shows the temporal evolution of $q_{0}$ in a comparable experiment with $2.5 \mathrm{MW}$ of applied ECCD. In qualitative agreement with simulation, $\mathrm{q}_{0}$ increases from an initial value of $\sim 4.8$ to a maximum value of $\sim 6.2$ in $\sim 1.0 \mathrm{~s}$. Evolution in a target equilibrium with neutral beam injection (NBI) alone shows no such change in $q_{0}$, in both simulation and experiment. Similar agreement between simulation and experiment is seen in the evolution of the minimum $q, q_{\min }$.
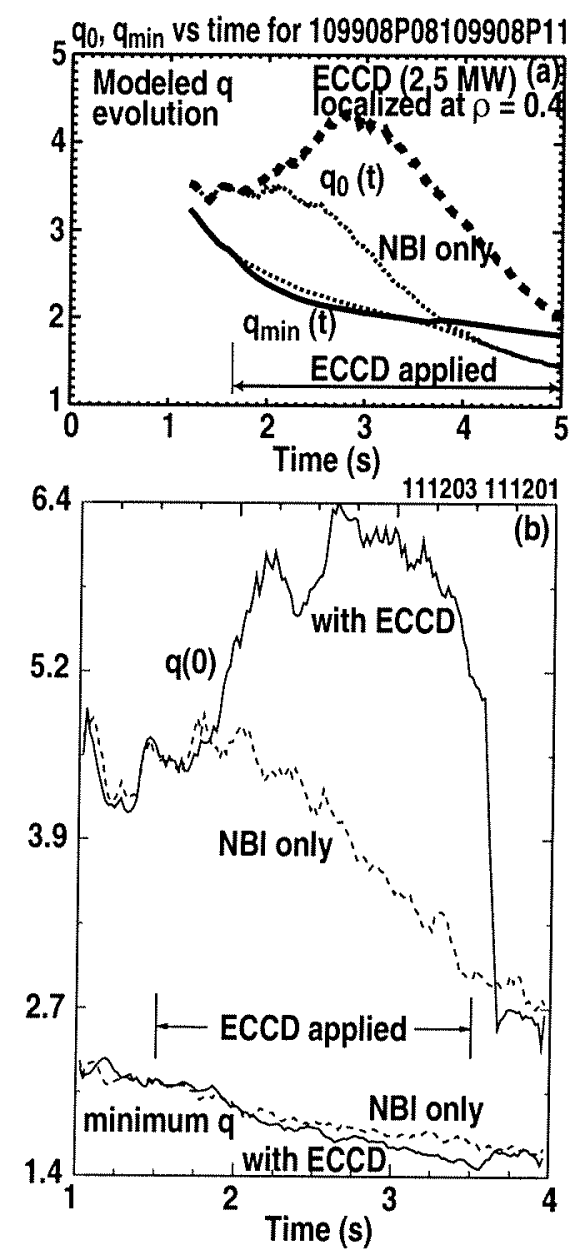

Fig. 1. Modification of the central safety factor by application of off-axis ECCD. 


\section{MHD STABILITY CONTROL}

A key characteristic of the AT regime is operation at high values of normalized beta above the no-wall limit at high bootstrap current fractions. The presence of large bootstrap current densities can make AT plasmas susceptible to NTM's [3]. Control of the current profile may allow maintenance of $\Delta^{\prime}$ sufficiently negative near rational $q$-surfaces to stabilize NTM's. Another approach used successfully on DIII-D is active suppression of the NTM by localized deposition of electron cyclotron current drive at the island location. Because real-time measurement of the island location is not yet available on DIII-D, the PCS implements a "search and suppress" algorithm to vary either the plasma position (and thus the island position relative to a fixed deposition region) or the toroidal field (and thus the deposition location relative to a fixed island location) in order to achieve the optimal island-ECCD alignment. The degree of alignment is inferred from measurements of the mode amplitude and its rate of change from fast Mirnov probe signals. A sophisticated nonlinear algorithm assesses the degree of alignment and determines the direction in which to search. Application of this algorithm typically produces complete suppression of the $3 / 2$ NTM within $500 \mathrm{~ms}$ of enabling the control phase. Figure 2 shows an example of this suppression, in which the control phase is enabled at $3.0 \mathrm{~s}$, simultaneously with $1.8 \mathrm{MW}$ of ECCD power. The search and suppress algorithm varies the plasma major radius in steps to determine the point of optimal alignment. This action can be seen in Fig. 2(b), in which the plasma major radius is moved outward in $1 \mathrm{~cm}$-steps as the mode amplitude decreases steadily [Fig. 2(a)]. In this case the mode is completely suppressed in $400 \mathrm{~ms}$ and remains suppressed until the control is disabled at $4.5 \mathrm{~s}$ and the plasma major radius returns to its original value (detuning the $q=3 / 2$ surface away from the ECCD deposition region). The 2/1 NTM was also completely suppressed in DIII-D this year using ECCD with the search and suppress algorithm, varying the toroidal field rather than the major radius.

Another MHD instability which limits access to high $\beta_{\mathrm{N}}$ is the RWM [4]. This mode is a low-m number ideal external kink whose growth rate is reduced from the ideal Alfvén frequency to approximately the resistive wall field penetration rate. The principal activator for RWM control in DIII-D is the correction coil (C-coil), a 6-element midplane toroidal array of saddle coils, typically connected in three $n=1$ pairs. The RWM can be stabilized through active feedback regulating the current in the $\mathrm{C}$-coil elements in response to magnetic measurements reflecting the $n=1$ mode amplitude. Figure 3 illustrates the effectiveness of this control action to allow increase of $\beta_{\mathrm{N}}$ above the no-wall $\beta$-limit while maintaining plasma stability. The case shown in red shows a large-amplitude RWM growing in the absence of feedback (causing $\beta_{N}$ to 

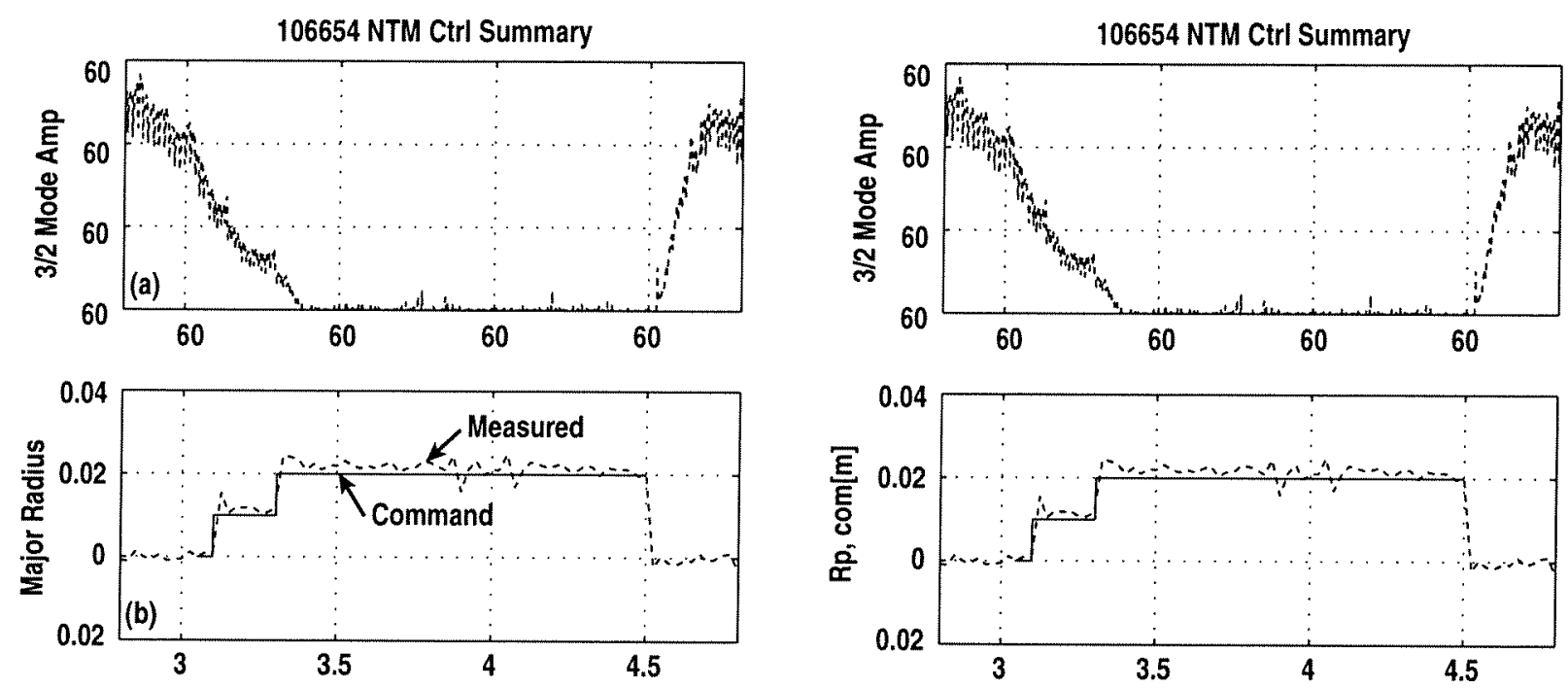

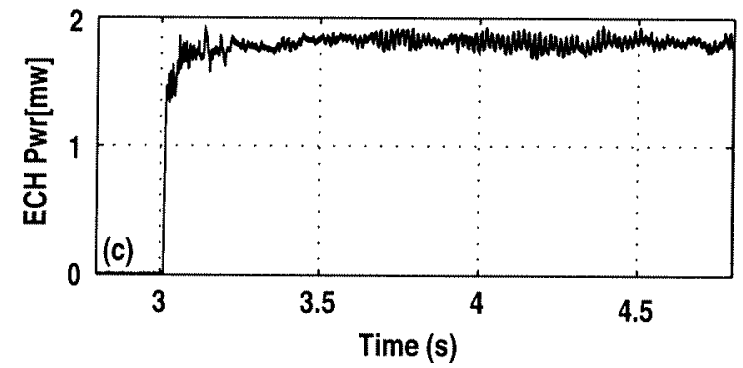

Fig. 2. 3/2 NTM control using major radial motion search and suppress algorithm.

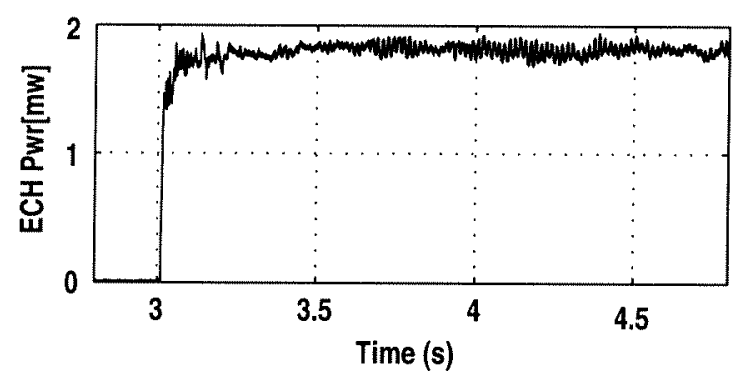

Fig. 3. RWM control through active feedback on measurement of $n=I$ mode.

decrease). In a comparison shot forming the same target equilibrium but with feedback enabled (shown in black), the mode is suppressed even though $\beta_{\mathrm{N}}$ remains high. 


\section{DETECTION AND MITIGATION OF IMPENDING DISRUPTIONS}

Thermal and electromagnetic damage from unmitigated disruptions pose a significant threat to tokamaks during operating point excursions which result in uncontrollable MHD instability. Although tokamak reactors will operate with sufficient control margin to limit the occurrence of disruptions, designs must provide protection for such off-normal events.

A method for mitigating these disruption effects using massive injection of noble gases (He, $\mathrm{Ne}$, or Ar) has been demonstrated on the DIII-D tokamak [5]. A jet of high injected gas density $\left(>10^{24} \mathrm{~m}^{-3}\right)$ and pressure $(>20 \mathrm{kPa})$ penetrates the target plasma at the gas sound speed $(\sim 300-$ $500 \mathrm{~m} / \mathrm{s}$ ) and increases the atom/ion content of the plasma by a factor of $>30-50$ in several milliseconds. UV line radiation from the impurity species distributes the plasma energy uniformly on the first wall, reducing the thermal load to the divertor by a factor of 10. Runaway electrons are completely eliminated by the large density of free and bound electrons, and the highly resistive post-jet plasma produces a significant $(>75 \%)$ reduction in peak halo current amplitude and attendant forces.

Use of this and other impurity injectionbased disruption mitigation methods requires sufficiently reliable detectors for impending disruptions. Figure 4 illustrates the use of a vertical position threshold detector to identify an intentionally-induced VDE and trigger the mitigation system. Following disabling of vertical control, the plasma moves downward and crosses the specified threshold vertical

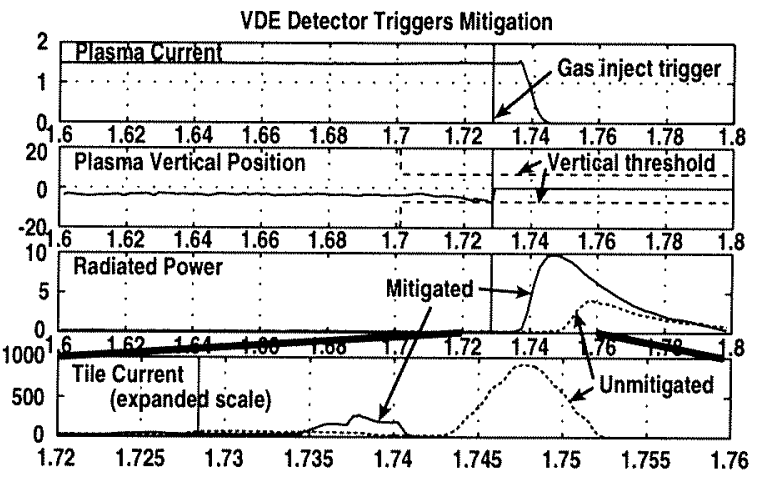

Fig. 4. VDE threshold disruption detection and mitigation with impurity gas injection. position $( \pm 5 \mathrm{~cm})$. The PCS triggers injection of high pressure Ne, producing a radiative thermal quench $\sim 4 \mathrm{~ms}$ after the trigger. Injection of the massive amount of Ne results in virtually all of the plasma thermal and magnetic energy being radiated, while the unmitigated case produces much less radiation (most of the thermal energy being conducted to the local wetted area of the wall). The gas injection also produces a colder post-thermal quench plasma, reducing the driven poloidal halo current by a factor of 4 . The corresponding local vessel pressure is reduced by a factor of 8 since the toroidal peaking is reduced by a factor of 2 by mitigation. Other detection algorithms implemented in the PCS include a radiated power threshold detector and a sophisticated NTM and locked mode detector. 


\section{SUMMARY AND CONCLUSIONS}

Many of the elements required for sustained achievement of the advanced tokamak regime have been demonstrated on the DIII-D tokamak. These include accurate plasma boundary control with simultaneous stored energy and density regulation, stabilization of the RWM and suppression of NTM's, and detection and mitigation of impending disruptions. While significant physics milestones remain, a principal focus of ongoing control efforts on DIII-D will be increased integration of operating regime regulation and MHD stability control, including profile control and improved off-normal condition detection and corrective responses. 


\section{REFERENCES}

[1] D.A. Humphreys, et al., Proc. 18th Symp. on Fus. Technol., Karlsruhe, Germany, August 1994, 1 (Elsevier Science Publishers B.V., Amsterdam, 1995) 731.

[2] J.R. Ferron, et al., Nucl. Fus 38 (1998) 1055.

[3] R.J. LaHaye, et al., Phys. of Plasmas 9 (2002) 2051.

[4] A.M. Garofalo, et al., Phys. Plasmas 6 (1999) 1893.

[5] D.G. Whyte, et al., "Mitigation of Tokamak Disruptions Using High-Pressure Gas Injection," accepted for publication in Phys. Rev. Letters, June 2002. 


\section{ACKNOWLEDGMENT}

Work supported by U.S. Department of Energy under Contracts DE-AC03-99ER54463, DEAC05-00OR22725, DE-AC02-76CH03073, and Grants DE-FG02-89ER53297 and DE-FG0395ER54294. 